\title{
Corrigendum
}

\section{Corrigendum to "Mucinous Histology, BRCA1/2 Mutations, and Elevated Tumor Mutational Burden in Colorectal Cancer"}

\author{
Noa Harpaz, ${ }^{1}$ Yair Eli Gatt, ${ }^{2}$ Roy Zvi Granit, ${ }^{1}$ Hila Fruchtman, ${ }^{3}$ Ayala Hubert, ${ }^{1}$ \\ and Albert Grinshpun $\mathbb{1}^{1}$ \\ ${ }^{1}$ Sharett Institute of Oncology, Hadassah-Hebrew University Medical Center, Jerusalem, Israel \\ ${ }^{2}$ Department of Microbiology and Molecular Genetics, Institute for Medical Research Israel-Canada, Faculty of Medicine, \\ The Hebrew University of Jerusalem, Jerusalem, Israel \\ ${ }^{3}$ Radiology Department, Hadassah-Hebrew University Medical Center, Jerusalem, Israel \\ Correspondence should be addressed to Albert Grinshpun; albert.grinshpun@mail.huji.ac.il
}

Received 6 July 2020; Accepted 7 July 2020; Published 11 September 2020

Copyright (C) 2020 Noa Harpaz et al. This is an open access article distributed under the Creative Commons Attribution License, which permits unrestricted use, distribution, and reproduction in any medium, provided the original work is properly cited.

In the article titled "Mucinous Histology, BRCA1/2 Mutations, and Elevated Tumor Mutational Burden in Colorectal Cancer" [1], some information was omitted in the Acknowledgments section, which should be corrected as follows:

\section{Acknowledgments}

This study was kindly supported by the Ministry of Science and Technology Personalized Medicine Grant. Y.E. Gatt was partially supported by the Hoffman Program. The authors would like to thank T. Bdolah-Abram from the Hebrew University of Jerusalem for her help with the statistical analysis. The study was conducted as part of the requirements for a Noa Harpaz's M.D. certificate in the Faculty of Medicine, the Hebrew University of Jerusalem.

\section{References}

[1] N. Harpaz, Y. E. Gatt, R. Z. Granit, H. Fruchtman, A. Hubert, and A. Grinshpun, "Mucinous histology, BRCA1/2 mutations, and elevated tumor mutational burden in colorectal cancer," Journal of Oncology, vol. 2020, Article ID 6421205, 10 pages, 2020. 